\title{
POSSIBILE APPLICATIONS OF MAGNETIC MICROWIRES IN AVIATION
}

\author{
Katarína Draganová \\ Josef Blažek \\ Dušan Praslička \\ František Kmec
}

Department of Aviation Technical Studies, Faculty of Aeronautics, Technical University of Kosice, Rampova 7, 04121 Kosice, Slovakia

\begin{abstract}
Magnetic microwires have been rediscovered due to a number of the unusual magnetic properties and their potential applications. The paper concerns glass-coated magnetic microwires composed of a ferromagnetic metallic core with a diameter of $0.6-30 \mu \mathrm{m}$ and of a glass coat with a thickness of $2-20 \mu \mathrm{m}$. The fabrication process and magnetic properties of these microwires are described. Due to their unique properties microwires can be used as a sensing element of sensors. Microwire-based sensors can be used in a wide range of aviation applications as magnetic field sensors, tensile stress sensors or temperature sensors. The main advantages of microwire-based sensors are associated with their small dimensions and weight, which play a very important role in aviation.
\end{abstract}

Keywords: Aviation, Magnetic microwires sensors, Magnetic field sensors, Mechanical stress sensors, temperature sensors

\section{INTRODUCTION}

Although studies dealing with magnetic microwires started in the 1970s, it was during the last decade that extensive research and progress in this area has been achieved. Magnetic microwires have been rediscovered mainly due to a number of the unusual magnetic properties and their potential applications. One possible use of magnetic microwires is as a sensing element of microwire-based sensors. Due to their weight, dimensions and multiple utilization possibilities micro-wire sensors can be used in many technical applications, aviation being no exception.

\section{MICROWIRES}

There are many types of microwires that have been developed and studied [1]. This paper is concerned with glass-coated magnetic microwires composed of a ferromagnetic metallic core with a diameter of $0.6-30 \mu \mathrm{m}$ and of a glass coat with a thickness of $2-20 \mu \mathrm{m}$ (Fig. 1). 


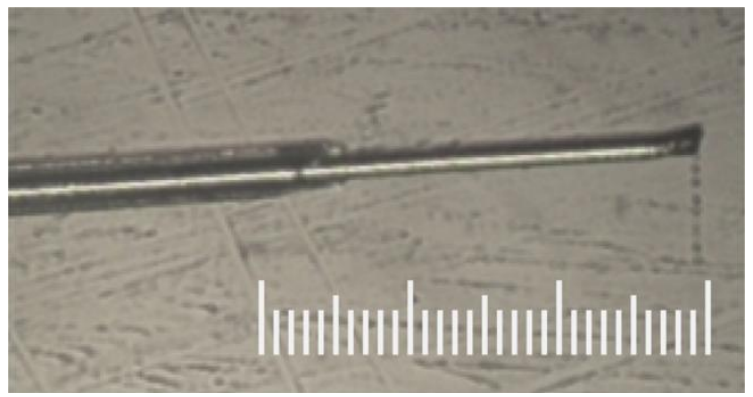

Fig. 1. Glass-coated microwire

\section{Fabrication process}

The most common fabrication technique of glass-coated microwires is the Taylor-Ulitovsky method [2] based on direct casting from the melt. What is important is that this fabrication technique allows us to control the microstructure and geometrical characteristics (diameter of nucleus and thickness of coating) of the microwire resulting in different mechanical, chemical and electrical properties. Another factor that strongly influences these properties is the chemical composition of the metallic core and the core and coat dimensions.

The fabrication process (Fig. 2) starts with putting several grams of an alloy with a desired chemical composition into a glass tube. The alloy is then heated up to its melting point using highfrequency induction heating. While the metal melts, the glass tube adjacent to the melting metal softens; subsequently the glass capillary is drawn from the softened glass portion and wound on a rotating coil. Under the right drawing conditions, the molten metal fills the glass capillary and a microwire composed of a metal core coated with a glass shell is formed. The microstructure of a microwire (and its properties) largely depends on the cooling rate, which can be controlled by a cooling mechanism when the metal-filled capillary enters into a stream of the cooling liquid (water or oil) on its way to the receiving coil. Special attention must be paid to the parameters that determine the critical quenching rate and cooling of the metal core so that the properties of microwires are reproducible.

The main advantages of this method of microwire fabrication are: repeatability of microwire properties in mass production, a wide range of variation in parameters (inner core diameter, glass thickness, physical properties), and the possibility to fabricate continuous pieces of microwire up to $10 \mathrm{~km}$ long. Finally, it is important to note the low price of microwires: $1 \mathrm{~kg}$ of alloy is sufficient to make $40,000 \mathrm{~km}$ of microwire, with the fabrication rate of several hundred meters per minute.

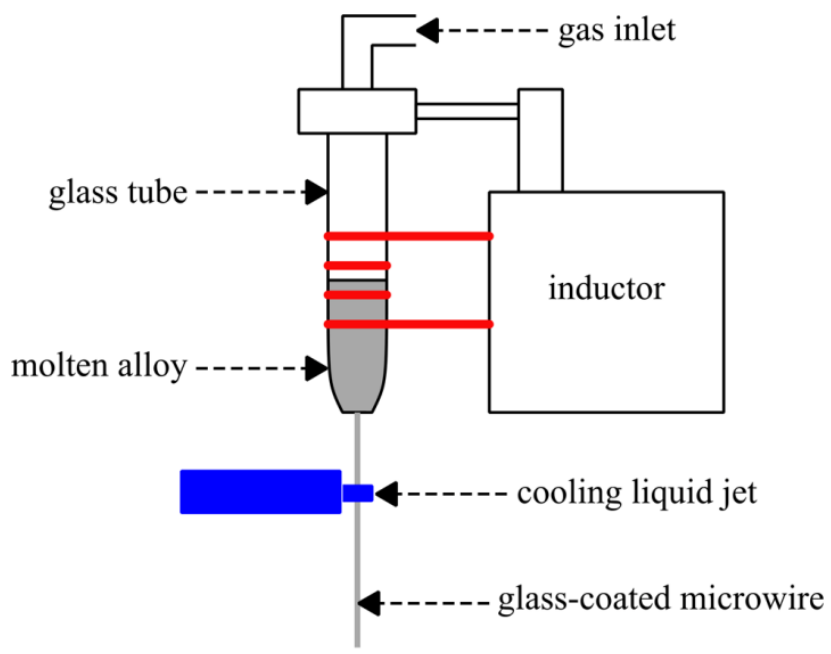

Fig. 2. Fabrication process 


\section{Magnetic properties of microwires}

Magnetic properties and magnetic behaviour of magnetic microwires are determined by their shape and by the magnetoelastic anisotropy arising from the coupling between internal stresses and magnetostriction. Magnetic properties such as coercivity, switching field, magnetic anisotropy field and magnetic permeability strongly depend on the microwire composition. The present research is focused on the amorphous Fe-based magnetic microwires with a high positive magnetostriction constant that are characterized by bistable behaviour and rectangular hysteresis loops (Fig. 3).

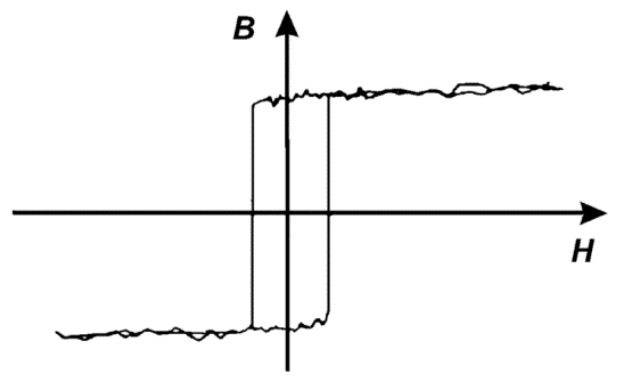

\section{Fig. 3. Hysteresis loop of a bistable amorphous microwire with a high positive magnetostriction constant}

The domain structure of microwires consists of one axial magnetic domain in the middle of the wire, whose cover is formed by radial domains. Closure domains are at the end of the wire. This structure causes the already mentioned bistable behavior resulting in magnetization having only two values and the switching between the two states running through the single Backhouse jump at the value of the field called the switching field. This field carries information about a number of physical quantities such as external magnetic field, tensile stress, temperature etc.

\section{MICROWIRE-BASED SENSOR}

\section{Theory}

Due to their unique properties microwires can be used as a sensing element of sensors. The sensor principle is based on the measurement of the switching field $H_{S W}$. It is the value of the field at which one magnetic field is switched to another and which can be calculated as a sum of the ambient magnetic field $H_{0}$ and the critical switching filed $H_{C S W}$ of the microwire:

$$
\boldsymbol{T} \boldsymbol{T} \boldsymbol{T} \boldsymbol{T} \boldsymbol{Z}_{\mathrm{s}}
$$

Apart from the magnetoelastic contribution $H_{S W}^{\sigma}$ to the critical switching field $H_{C S W}$, there exists another contribution that arises from the structural relaxation of amorphous microwires $H_{S W}^{r}$ :

$$
\text { TESTFWTH }
$$

Magnetic properties predetermine microwire-based sensors for utilization as magnetic sensors. But due of the fact that the magnetic properties of amorphous glass-coated microwires are also determined by the magnetoelastic interaction between the magnetic moment and the mechanical stresses, the switching field is very sensitive to mechanical stresses applied, too. The measured tensile stress dependence is shown in Figure 4 (left). Furthermore, due to different thermal expansion coefficients of the metallic nucleus and the glass coating, additional stresses are applied to the metallic core, causing the switching field to be sensitive to temperature, as can be seen in Figure 4 (right). 

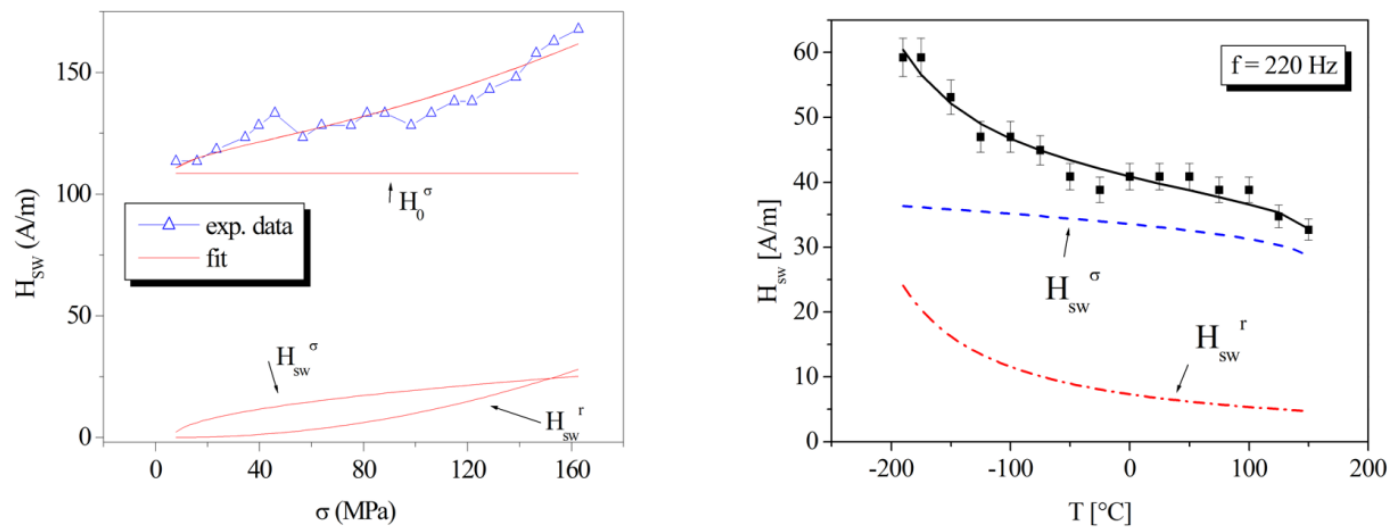

Fig. 4. Tensile stress and temperature dependence of $\mathrm{Fe}_{73} \mathrm{Nb}_{3} \mathrm{Si}_{11} \mathrm{~B}_{13}$ microwire [3][4]

\section{Measurement method}

For the determination of the switching field, an induction method was used [5]. When the excitation coil is fed by a precision triangular shape current, the switching field is proportional to the time in which the domain wall's motion occurs. The time shifts between the excitation magnetic field and the induced voltage peaks that represent the domain wall's motion allowed researchers to determine the switching field. Peaks were detected using the sensing coil. The microwire can be placed either inside the sensing coil or outside the sensing system. This improvement made it possible to create a contactless sensing system.

\section{POSSIBLE APLICATIONS OF MICROWIRES IN AVIATION}

Microwire-based sensors can be used in a wide range of aviation applications as magnetic field sensors, tensile stress sensors or temperature sensors. The main advantages of microwire-based sensors are associated with their weight, dimensions and low manufacturing costs which makes them very suitable also for utilization on small unmanned aerial vehicles (UAVs). An example of the placement of selected types of microwire-based sensors is shown in Figure 5.

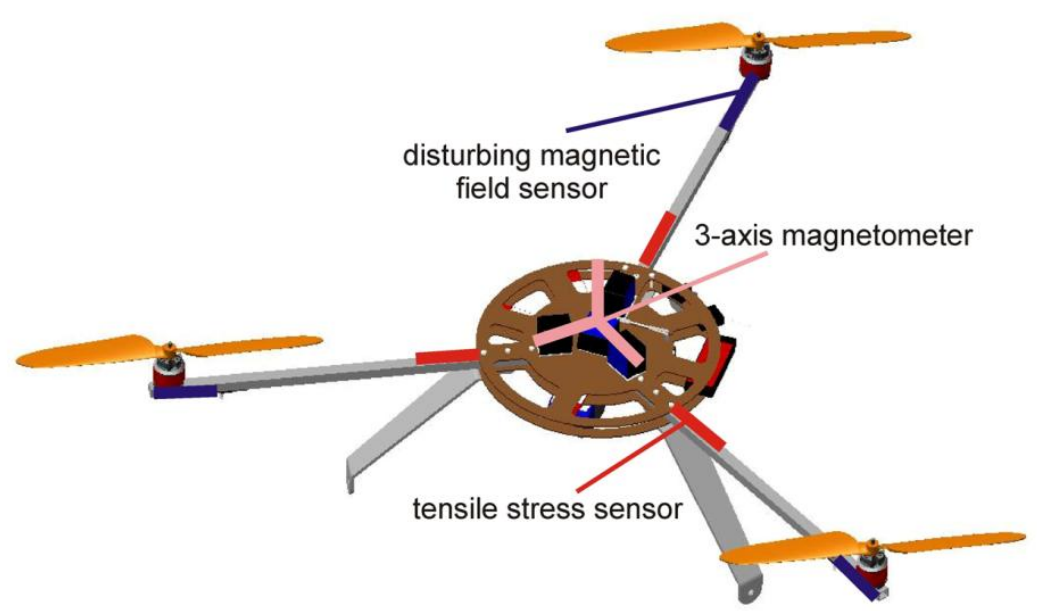

Fig. 5. Microwire-based sensor utilization in UAVs 


\section{Magnetic field sensors}

Magnetic field sensors can be used for example for navigation purposes for heading determination. They can replace or at least aid the existing compasses. Another possible application of magnetic sensors is in inertial measurement units, where they can be used as inertial sensors together with an accelerometer and gyroscopes.

Given the fact that other sensors can be sensitive to disturbing magnetic fields, microwire-based sensors can be used for monitoring the disturbing magnetic fields arising for example from electrical sources, power plants and electric cables placed on boards. These outputs can be consequently used to compensate the mentioned disturbing magnetic fields.

Another way how to use sensing of current flowing through the wires might be to determine electric power consumption or to calculate the characteristics of a power plant's performance.

\section{Temperature sensors}

Microwire-based sensors can be used as temperature sensors for example for environmental or meteorological purposes. Temperature sensors may be also used for temperature dependence compensation due to the fact that many types of sensors are characterized by cross-sensitivity and temperature dependence is not negligible.

\section{Tensile stress sensors}

Microwire-based tensile stress sensors offer great promise for example for aircraft construction monitoring and structural health monitoring. Also, operational load can be monitored with this type of sensors. These sensors can also prove their worth in fatigue testing or crack detection. And when using the improved induction method developed by the present authors, sensing can be absolutely contactless. This fact in conjunction with small dimensions of microwires offers the possibility of integrating microwires directly into the material. Microwires can be easily built into many different types of structures without causing structural damage or adversely affecting material characteristics.

Alongside its many advantages, the method has one limitation - sensors can be only used inside of nonmagnetic materials.

\section{CONCLUSIONS}

Amorphous magnetic microwires are relatively novel materials that due to their unique properties can be used in a wide range of applications. Many types of microwires of different characteristics have been developed and manufactured using different fabrication techniques. This contribution deals with bistable glass-coated amorphous magnetic microwires and their utilization in sensorics where they can serve as a sensing element of a sensor.

Magnetic microwires are primarily used for magnetic field sensing. In aviation applications, they can be used for navigation purposes for heading determination or as magnetometers in inertial measurement units. Also, magnetic sensors make it possible to compensate disturbing magnetic fields. But due to the fact that microwires are sensitive also to some other physical quantities, they can be used for example as temperature sensors for temperature monitoring or compensation. As tensile stress sensors, magnetic microwires can be used in aircraft construction, structural health and operational load monitoring, fatigue testing and crack detection. 
The main advantages of microwire-based sensors are associated with their small dimensions and weight, which play a very important role in aviation. Low manufacturing costs also present a considerable advantage, especially for utilization on small unmanned aerial vehicles. In the area of material testing and monitoring, contactless sensing and so non-destructing testing and material defect detection are key factors that predetermine microwires for utilization as microwire-based tensile stress sensors.

\section{ACKNOWLEDGEMENT}

This work was supported by the Slovak Research and Development Agency under Contract No. APVV 0266-10.

\section{REFERENCES}

[1] Handbook of Magnetism and Advanced Magnetic Materials. Editors KRONMULLER, H., PARKIN, S. New York : Wiley-Interscience, 2007. ISBN 0470022175.

[2] LARIN, V.S., TORCUNOV, A.V., ZHUKOV, A., GONZÁlEZ, J., VAZQUEZ, M., PANINA, L. Preparation and Properties of Glass-Coated Microwires. In Journal of Magnetism and Magnetic Materials, 249 (2002), p. 39-45.

[3] PRASLIČKA, D., BLAŽEK, J., ŠMELKO, M., HUDÁK, J., ČVERHA, A., MIKITA, I., VARGA, R., ZHUKOV, A. Possibilities of Measuring Stress and Health Monitoring in Materials Using Contact-Less Sensor Based on Magnetic Microwires. In IEEE Transactions on Magnetics, Vol. 49, No. 1, January 2013.

[4] VARGA, R., GARCIA, K. L., ZHUKOV, A., VAZQUEZ, M., VOJTANIK, P. Temperature dependence of the switching field and its distribution function in Fe-based bistable microwires. In Applied Physics Letters, 83, 2620, 2003.

[5] BLAŽEK, J., PRASLIČKA, D., ŠMELKO, M., DRAGANOVÁ, K., HUDÁK, J., MIKITA, I., LIPOVSKÝ, P., VARGA, R. Contribution to Modern Methods of Rapid Testing of ElectroMagnetic Properties of Metallic Materials. In: Physics of Materials 2012 : Proceeding of the Scientific Conference. Košice : TU, 2012. P. 1-5. ISBN 978-80-553-1175-3. 